\title{
First Person Shooters on the Road: Leveraging on APs and Vanets for a Quality Gaming Experience
}

\author{
Jose Saldana \\ Communication Technologies Group (GTC) \\ University of Zaragoza \\ Zaragoza, Spain \\ jsaldana@unizar.es
}

\author{
Gustavo Marfia, Marco Roccetti \\ Department of Computer Science \\ University of Bologna \\ Bologna, Italy \\ \{marfia, roccetti\}@cs.unibo.it
}

\begin{abstract}
This work studies the feasibility of using on the road 802.11 Access Points (APs) for the support of First Person Shooter (FPS) games, which are among the online services that exhibit the tightest real-time requirements nowadays. After demonstrating that an acceptable quality of service can be guaranteed, we propose and assess the extension of the AP coverage area by means of vehicular ad-hoc (VANET) communications. The time for which a game can be played in a satisfactory manner can be, in fact, increased exploiting neighboring vehicles, thus improving the general gaming experience. To this aim, we adopted a subjective quality estimator, which reveals whether a game could, or could not, be properly run in a given scenario. Our simulation results show that the AP coverage area can be significantly increased when using other cars as packet forwarders, while maintaining the subjective quality at acceptable levels. All this is performed assessing the feasibility of FPSs on realistic vehicular traffic traces, obtained recording over 100 traversals of an urban road under varying traffic conditions.
\end{abstract}

Keywords-inter-vehicular communications; online games; realtime services; Quality of Experience; First Person Shooter

\section{INTRODUCTION}

In the past few years, mobile devices have gained an increasingly important role in the computer gaming arena. More and more mobile customers, in fact, fill up their idle intervals of time (e.g., on the bus, going to work) playing some game while on the move. The vast majority of such games is either individual or turn-based, thus do not critically depend on network latency. Hence, for such type of games, the role played by wireless connections is limited to providing a fast download of the desired app. However, interactive multi-player games, as First Person Shooters (FPSs), racing games or Massively Multiplayer Online Role Playing Games (MMORPGs), are also expected to reach a high share of users in mobile scenarios in the next few years [1]. Many of such games are based on client-server architectures, where game providers deploy and maintain servers equipped with adequate levels of bandwidth and processing power. Nevertheless, the gaming quality experienced by a given player strongly depends on the performance of the connection set up between his/her device and the server that his/her handheld joined.

This work has been partially financed by CPUFLIPI Project (MICINN TIN2010-17298), MBACToIP Project, of Aragon I+D Agency and Ibercaja Obra Social, NDCIPI-QQoE Project of Catedra Telefonica, Univ. of Zaragoza, and Programa Europa, from CAI and Gov. of Aragon (IT 17/11).
Interestingly, the use of mobile devices is also expected to become popular within the context of moving vehicles, as more and more vehicles will become equipped with 802.11 interfaces, and be able to support Internet connections through vehicular-to-vehicular (V2V) and vehicular-to-infrastructure (V2I) communications. In fact, although the 802.11 standard was not initially designed for supporting communications on moving vehicles, this technology is being adopted to also support such type of scenarios [2], [3], [4]. Hence, many research initiatives have proposed applications based on the use of $\mathrm{V} 2 \mathrm{~V}$ and V2I communication paradigms. Among these, relevant streams of work have investigated how accident prevention applications, as well as traffic management ones, could put to good use the exchange of messages between cars [5]. None of these, however, require the support of real-time streams between a moving vehicle and a server.

Now, if vehicular networks will become true, we can envision future situations where passengers (e.g., kids in the back seat) will pass their time engaged with their mobile devices while connected to the Internet, playing their favorite FPS or MMORPG games against other players that are connected from elsewhere to the same gaming arena.

Clearly, all this will be possible only if an adequate quality of service will be provided to those mobile players. In order to verify the feasibility of such scenario, we here consider two, apparently unrelated, problems: on one hand, real-time requirements of online games, on the other hand, the use of wireless infrastructures for providing connectivity to moving vehicles. Hence, a first contribution of this work is a feasibility study that assesses the use of 802.11 for the provision of satisfactory levels of quality of service to multi-player games inside moving cars. Provided this possibility, the second and most relevant contribution of this work is the support of realtime services through the use of $\mathrm{V} 2 \mathrm{~V}$ connections that extend the coverage area provided by APs: if a WiFi-enabled car is inside AP coverage, it serves as a forwarding relay to and from those neighboring vehicles that fall outside. In fact, the enlargement of the coverage area translates into a longer connection time, thus improving the gaming experience. This is the scope of this work: we explore the feasibility of providing satisfactory quality of service levels to real-time applications, such as FPS games, in a vehicular scenario through the combination of V2I and V2V connections. Our contribution differs from any previous work, which exploited the use of "traditional" applications [2], [3], [6], e.g. file transfer or web 
browsing, as the requirements imposed by FPS games on the underlying network are way more stringent. This type of applications, in fact, are very challenging for wireless networks, since a high quality experience requires high levels of interactivity [7], thus implying tight limits for delay, jitter and packet loss.

To fulfill our analysis, we selected a FPS game as a relevant exemplar of a real-time application. In such game a given user controls an avatar whose mission is that of killing all other characters in a virtual scenario. Clearly, in such scenario, responsive movements, which generate fast paced inputs to the game, are very important. These generate considerable amounts of UDP packets per second (up to 85), but of small size (typically a few tens of bytes of payload) [8]. We tested such game running, in simulation, on a vehicular network, where vehicles moved with realistic running patterns along an urban road, as they were drawn from over 100 traversals performed with our cars in reality.

The rest of the paper is organized as follows: the next section talks about the related work. The scenario of interest is explained in Section III. Section IV shows the achievements and results, and the paper ends with the Conclusions.

\section{RELATED WORK}

Since we are studying the feasibility of 802.11 for real-time applications, in this section we will summarize the state of the art about these two problems: first, the use of 802.11 APs for providing connectivity on the road; second, the problem of estimating subjective quality for real-time applications.

\section{A. Using 802.11 in Vehicular Scenarios}

The performance of a WLAN for vehicular connectivity was measured in [9] for different scenarios. A connectivity range of 1,000 meters was achieved in some cases. In [2] the use of 802.11 APs for connecting moving vehicles was also studied. Three different phases were observed: connection, disconnection, and a "production" one, in which the data was mostly exchanged. The maximum throughput obtained was about 5 Mbps.

In [6], the authors explored the feasibility of communications between a moving vehicle and an 802.11based AP. The tested services were TCP bulk transfers, UDP flows and web browsing. Some of the tests included a backhaul network segment, in order to emulate the Internet. One of the conclusions was that network and application issues had a worse influence on the performance of the service than the wireless link itself. The effect of the backhaul network, mainly adding a 1 Mbps limit and a delay, was really harmful for web traffic, since the throughput diminished dramatically.

In [3] a system named Cabernet was proposed so as to deliver data to and from cars using $802.11 \mathrm{~b} / \mathrm{g}$ APs. The cause of using this access technology was that its bandwidth is bigger than the one of current cellular networks. The system was thought for applications using opportunistic connections, i.e. not needing a continuous exchange of data with a server (e.g. transmitting data from sensors, message delivery). Thus, it was proposed as a suitable complement to $3 \mathrm{G}$ and $4 \mathrm{G}$ networks: by setting a number of APs, spatial reuse of the same frequencies can provide a good connection method. In contrast, what we are studying in the present paper is the possibility and the conditions required for delivering a real-time service in the same scenario, using 802.11 APs next to the street or the road.

In [10] vertical handoffs in a scenario considering a cellular network in addition to a WLAN were studied. In addition, the authors considered the possibility of using V2V communications to extend the coverage of the WLAN.

A protocol for $\mathrm{V} 2 \mathrm{~V}$ communications was proposed in [11] in order to broadcast messages, mainly about traffic, weather or even advertisements. It used the predictability of the car's movements in order to improve the efficiency of channel utilization.

\section{B. Subjective Quality Estimators for Online Games}

The idea of obtaining an estimator of the QoE of a service, as a function of objective network parameters, was first developed for VoIP. The E-Model [12], proposed by the ITU, is able to generate an estimator of the expected Mean Opinion Score (MOS from now) of the service, which ranges from 1 (bad quality) to 5 (excellent). The input variables are network parameters (delay, packet loss), and also the codec and other factors modifying the voice quality. Logically, the model is developed from surveys in which real people are involved.

In [13] a similar quality estimator was developed for a FPS game $($ Quake $I V)$. Although the bandwidth of these games is small (some tens of kbps), they have very stringent real-time requirements. This quality model uses delay and jitter as input variables, since the game implements a very effective packet loss concealment method [14].

In this work we have decided to use a FPS game because it constitutes an example of a service with really tight real-time requirements, in which delays above $150 \mathrm{~ms}$ may be intolerable by many users [14]. In addition, the use of a subjective quality estimator makes the results more interesting, since we not only obtain the objective parameters from the network, but we also integrate them into a single figure estimating the user's experience with the service.

\section{SCENARIO OF INTEREST: MODELING AND SIMULATION}

As reported in [3], 802.11 APs are being used in some cities as a means for connecting to the Internet. The performance of $3 \mathrm{G}$ and $4 \mathrm{G}$ networks and their high costs are still a barrier for their widespread use with all applications, as e.g. online games. What is interesting, instead, is that throughout the most urbanized areas, in the western world, many restricted access APs, but also many open ones, are densely distributed. The latter ones, hence, can be exploited for the provision of applications to customers on the move.

In fact, the first challenge we are trying to explore in this work is the possibility of using a multimedia application in this scenario. Thus, we will simulate a street with a single AP partially covering it (Fig. 1). We here select a $2 \mathrm{~km}$ long road: as shown in a recent study, it can be considered a typical length in an urban setting [15]. 


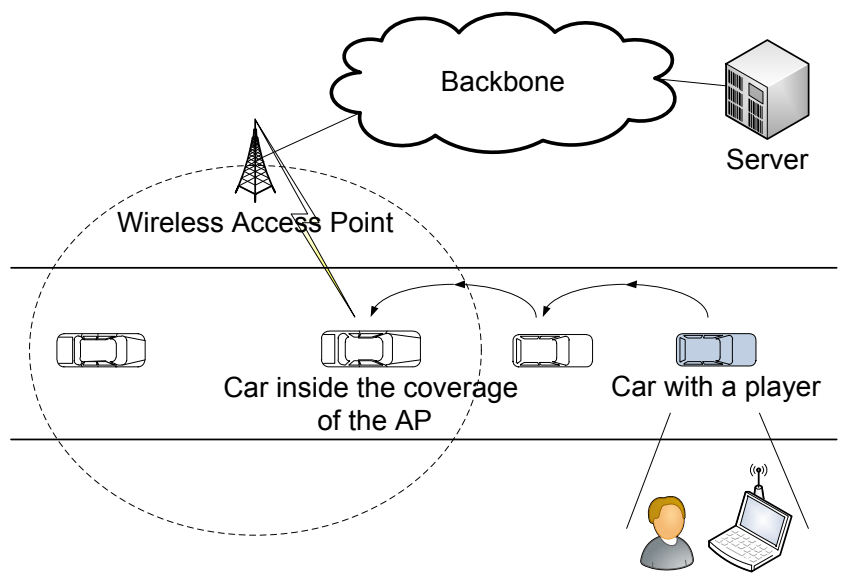

Figure 1. 802.11-enabled cars used for relaying packets to the AP

The second objective of the tests is exploring the feasibility of increasing the time a player can use a multimedia application, i.e. for how long a car out of AP coverage can keep using the application, until other cars forward its packets to and from the AP (Fig. 1). So, in this second scenario, other WiFienabled cars create an ad-hoc network. In this section we will describe the tools we have used to model the scenario and obtain the results.

\section{A. Simulation Settings}

An ns2 simulation scenario (Fig. 2) has been developed, including a street with an $802.11 \mathrm{AP}$, having direct visibility with the cars. Every second, a new car appears at the beginning of the street. There is a Fixed Node (FN), which simulates the game server, connected via the Internet to an AP. A hierarchical network address structure has been set in the simulator, in order to make the AP forward packets between the wired and the wireless networks. Some of the cars are running a FPS game, which consists of two UDP flows, one for the uplink and another one for the downlink. If the car running the application is outside the coverage area of the AP, other cars can forward the packets by the use of an ad-hoc routing algorithm. The default parameters of the simulations have been included in Table I.

\section{B. Generation of Game Traffic}

In order to generate realistic traffic, traces captured in real parties have been obtained from [16]. The ability of ns2 for sending traces from binary files has been used to generate the traffic exactly as it was in the real party. Each client sends 64 pps to the server, with an average size of 79 bytes ( $40.5 \mathrm{kbps})$. The server traffic corresponds to a 5 players' party, generating $14 \mathrm{pps}$ with an average size of 161 bytes (18 kbps).

\section{Calculation of the MOS}

As we have previously said, in [13] a subjective quality estimator (G-Model) was proposed for a FPS game (Quake IV), depending on delay and jitter. The model was obtained by deploying a number of parties with real players, while modifying the network in order to artificially introduce delay, jitter and packet loss. Surprisingly, packet loss did not affect below $35 \%$, so the authors decided to make the MOS only

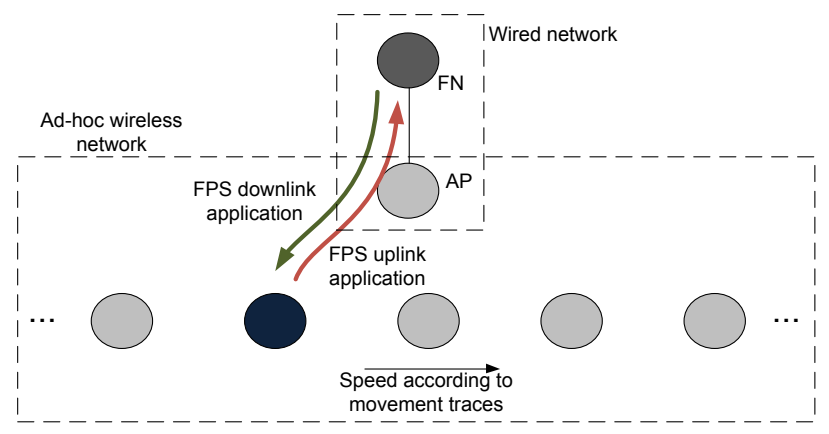

Figure 2. Scenario used in the simulations

TABLE I. SIMULATION PARAMETERS

\begin{tabular}{|l|c|}
\hline \multicolumn{1}{|c|}{ Parameter } & Value \\
\hline Propagation model & Two Ray Ground \\
\hline Street length & $2000 \mathrm{~m}$ \\
\hline Street width & $10 \mathrm{~m}$ \\
\hline Time between cars & $1 \mathrm{sec}$ \\
\hline Access Point and nodes coverage radius & $250 \mathrm{~m}$ \\
\hline Access Point position & $1000 \mathrm{~m}$ \\
\hline 802.11 rate & $54 \mathrm{Mbps}$ \\
\hline Ad-hoc routing algorithm & DSDV \\
\hline Update period of DSDV & $0.5 \mathrm{sec}$ \\
\hline One Way Delay Access Point-Fixed Node & $20 \mathrm{~ms}$ \\
\hline
\end{tabular}

depend on delay (measured as round trip time) and jitter (measured as the standard deviation of the delay). Multidimensional regression analysis was used so as to obtain the model. First, an impairment parameter $X$ is obtained:

$$
X=0.104 * \text { ping_average }+ \text { jitter_average }
$$

And the MOS, ranging from 1 to 5, is calculated using:

$$
\text { MOS }=-0.00000587 X^{3}+0.00139 X^{2}-0.114 X+4.37
$$

The value of the tick has been set to 1 second, i.e. we will present the MOS as the average value for all the packets received in that tick. When packet loss is above $35 \%$, we will consider MOS to have a value of 1 . Some works use the same MOS scale of VoIP, and consider it acceptable above 3.5. Nevertheless, some others consider that a value of 3 can be good [17], and gamers would exchange to another server when MOS is about 2.

\section{ACHIEVEMENTS AND Results}

In this section, we want to answer the next questions: First: Is it possible to have a good MOS for a FPS in this vehicular scenario? Second: How much can we extend the coverage area of the AP with a hybrid V2I-V2V network infrastructure? Third: What happens if we use real movement traces? Finally, we will study the effect of background traffic on the QoE of the game. 


\section{A. Is it possible to obtain a good MOS in the scenario?}

To answer this first question, we deployed a test, in which a single car running the game moved along the street at a constant speed of $50 \mathrm{~km} / \mathrm{h}$, while directly communicating with the AP. In Fig. 3 we present the MOS, which is always above 3.7. Although not shown in the figure, the delay is about $42 \mathrm{~ms}$, and jitter is roughly $0.5 \mathrm{~ms}$; both of them present a very constant behavior while the car is in the coverage area. The fact of the delay being of $42 \mathrm{~ms}$ means that its value is mainly determined by the fixed network $(40 \mathrm{~ms})$. This result demonstrates the first idea proposed in this work: an AP is able to provide acceptable communications quality for the applications of interest in the given scenario. Logically, this only happens while the car is inside its coverage area.

\section{B. Can we extend the coverage area while maintaining the MOS?}

Next, we want to test the feasibility of using V2V communications in order to extend the coverage area of the AP. For this purpose, we have deployed a simulation in which 300 cars go down the street, creating an ad-hoc network. A car running the FPS game appears in the street in the place number 150 , in order to have the street "filled with cars" when it arrives. Again, all the cars have a constant speed of $50 \mathrm{~km} / \mathrm{h}$. We present the results for a representative single run, which will help us understand the phenomena that occur in this scenario. Average values, as well as their corresponding confidence intervals will be presented in the next subsection.

Fig. 4 presents the MOS. From the figure, it can be seen that, as soon as the car enters the street, it starts receiving and sending packets by means of the VANET. Since the game uses UDP, it keeps on sending packets despite the fact that they are not received. In order to better understand the results, in Fig. 5 we have included (a) delay, (b) jitter, (c) packet loss and (d) the number of ad-hoc hops of downlink packets.

During the first stage (first 292 meters), the quality is not acceptable because the ad-hoc protocol keeps on modifying the best route, producing jitter and loss peaks, with intervals which are multipliers of 13.8 meters (the distance between cars). This can also be seen in Fig. 5 (d), where an oscillation in the number of hops can be observed. Above 300 meters, the delay caused by the hops, and the jitter peaks are reduced, so the MOS becomes acceptable until the car enters the coverage area.

Once the car gets out of the coverage area, a "handoff" can be observed, associated with an increase of the delay, caused by the time the ad-hoc protocol needs to find the new route. After that, a zone in which MOS is again acceptable can be observed. But, why does this "handoff" phenomenon not appear when the car enters the AP coverage? In fact, when the car passes from a situation with $\mathrm{V} 2 \mathrm{~V}$ connectivity to another one with direct AP connectivity (i.e., V2I), no packets get lost. Hence, once the car is in the AP range, it directly sends its packets to the AP. On the other hand, when the car leaves the AP coverage, some time is needed to readjust the routes of the ad-hoc routing protocol. Different routing algorithms may present different behaviors, but this is beyond the scope of the present work. In [18] different schemes for V2V and for V2I mobility management were discussed. However, the

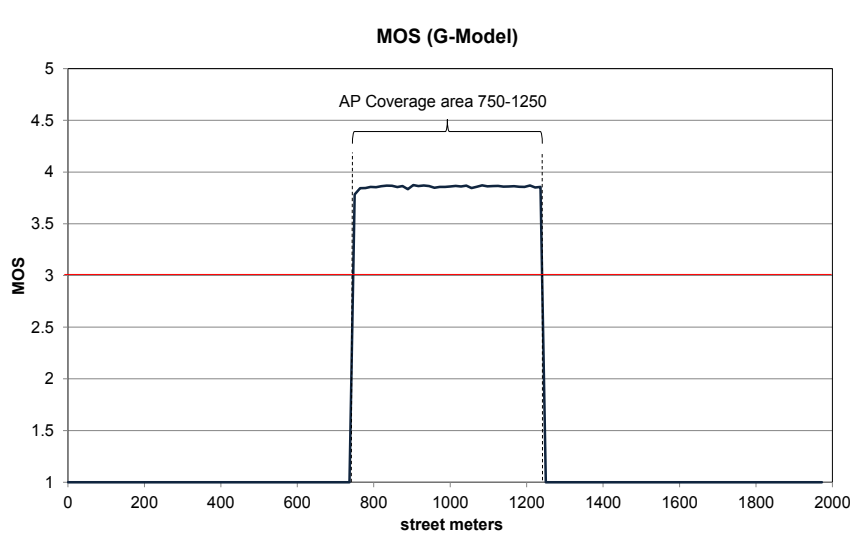

Figure 3. MOS for a single car, not using V2V communications

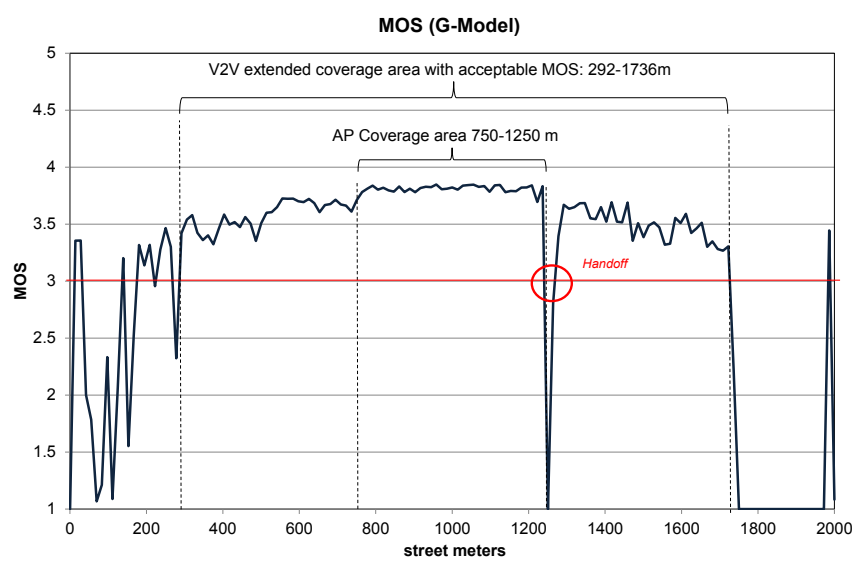

Figure 4. G-Model MOS for the V2V scenario

phenomenon we observe here is a sort of "V2V to V2I handoff", which may require specific algorithms in order to be correctly managed. A survey of routing protocols for these scenarios can be found in [19]. A cost and delay study of handoffs in a similar scenario can be found in [10].

All in all, the coverage area has been extended in this case from 500 to 1,444 meters $(288 \%)$ by the use of V2V communications.

\section{What if we make it even more realistic?}

The previous results have been obtained imposing a constant speed to all the cars. In this subsection we will consider a more realistic scenario in which the movements of the cars have been simulated using 111 traces obtained from a real car moving on Via Benedetto Croce, Pisa, Italy [20]. The traces have been divided into five groups, according to their average speeds, and each test is deployed using traces from one of the groups. Again 300 cars are sent, and car number 150 runs the game. Each car randomly selects movement traces of the group, thus allowing us to compare different traffic density situations. The average speed of the traces ranges from 8.4 to $29.1 \mathrm{~km} / \mathrm{h}$.

For each car, "contact time" is defined as the duration of the longest interval with acceptable MOS. Two "bad ticks" are tolerated, assuming that this corresponds to the "handoff". Fig. 


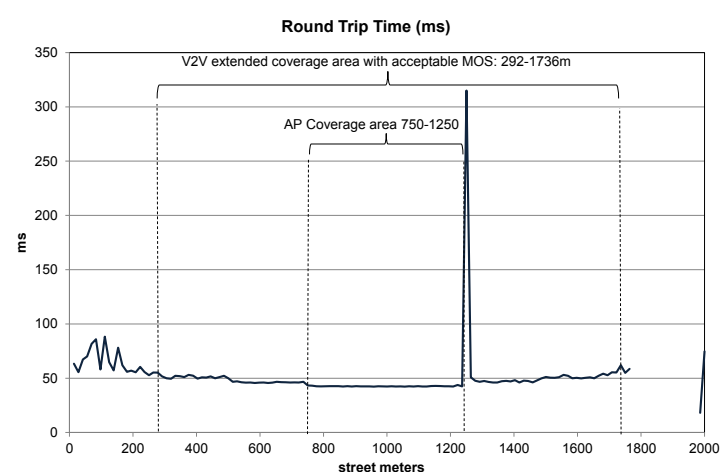

(a)

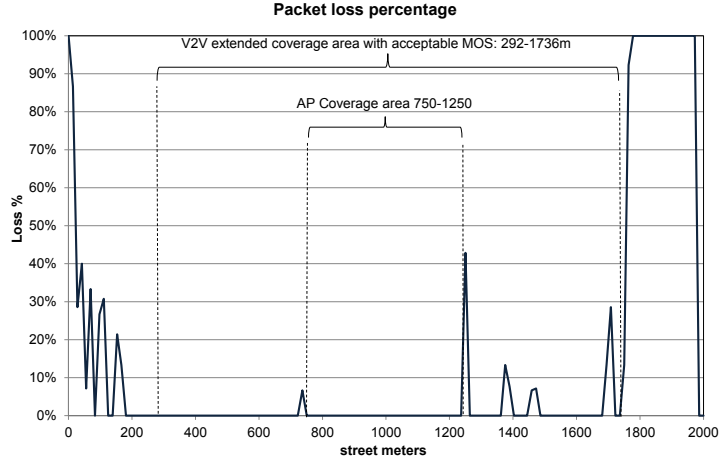

(c)

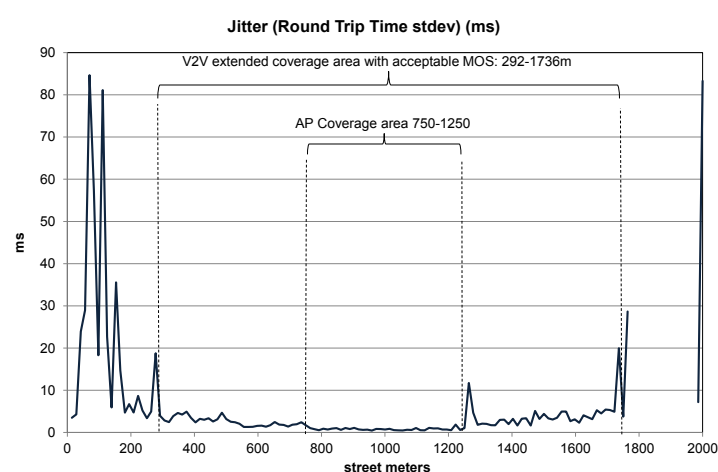

(b)

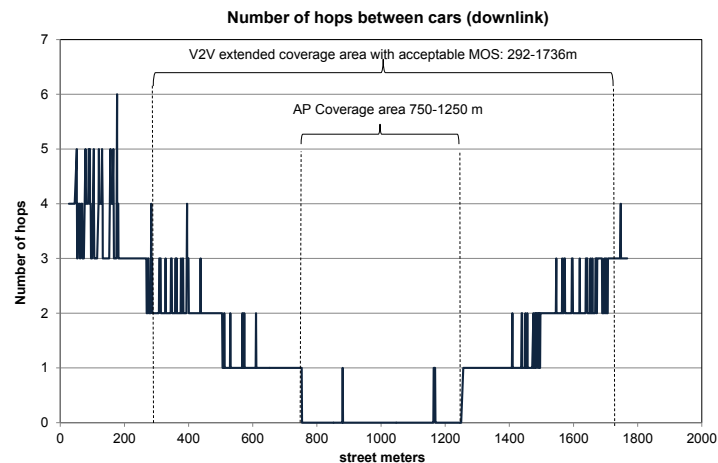

(d)

Figure 5. Scenario including V2V communications: a) Round Trip Time; b) Jitter (stdev of Round Trip Time); c) Packet Loss; d) Number of V2V hops

6 presents the average duration of the contact time, with and without the use of V2V. Each test has been repeated 10 times, and $95 \%$ confidence intervals are also presented. The value of the $\mathrm{X}$-axis is the average speed of the traces of each group.

It can be seen that the improvement achieved by the use of inter-vehicular communications is significant (in some cases the contact time is doubled), since the average time passes from the one corresponding to the AP coverage to an enhanced one. It can also be observed that the variation is smaller when no $\mathrm{V} 2 \mathrm{~V}$ is used, since the game only works while being in the coverage area of the AP.

\section{On the negative effect of TCP background traffic}

In this subsection we will study the effect of background traffic on the QoE of the game. By now, we have only considered cars running the FPS game. The next tests also include some cars running an FTP download.

A FTP download can heavily deteriorate gaming quality, since TCP increases its transmission window until a packet is lost, and this tends to produce a saturation of the available bandwidth. Different mechanisms for controlling TCP bandwidth have been proposed, in order to grant the feasibility of real-time services in this scenario: e.g. [21] studied the use of intelligent APs to limit the maximum value of the TCP advertised window.

We have done the same test run in IV.B, but this time, in addition to car number 150 running a FPS, the car that follows (car number 151) runs a FTP download, using TCP SACK. Fig. 7 (a) shows the throughput of the FTP application. Fig. 7 (b) and (c) present the MOS and the jitter of the FPS car

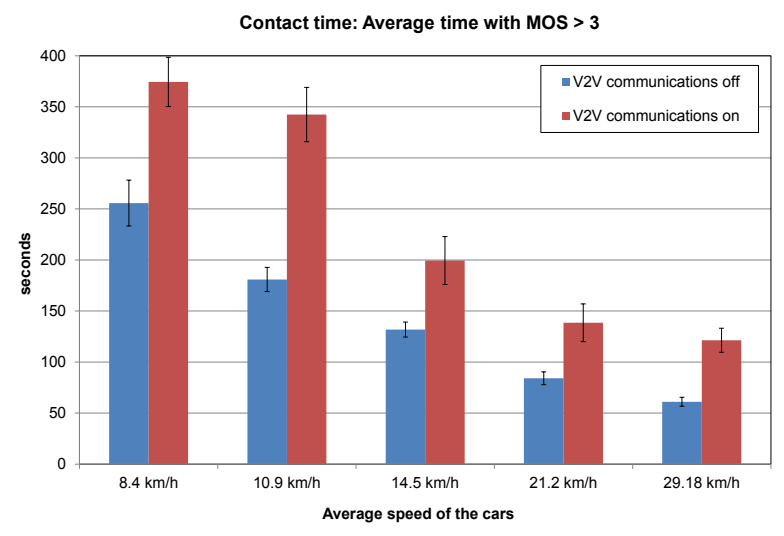

Figure 6. Average connection time with good quality

respectively. It can be seen that FTP jeopardizes the beneficial effect of the VANET, only permitting the game to be continuously played within the coverage area, although with a low quality.

\section{CONCLUSIONS}

This article has studied the feasibility of using 802.11 networks for providing connectivity to the use of FPS games. It has been shown that these networks are capable of providing a good subjective quality for real-time online gaming. In addition, the V2V communications have been used to extend the coverage area of an AP. Tests with real movement traces have been performed, in order to measure the increase of the time where the service can have a good quality, when using $\mathrm{V} 2 \mathrm{~V}$ communications. The results show that V2V can significantly increase the time that the game can be played with 


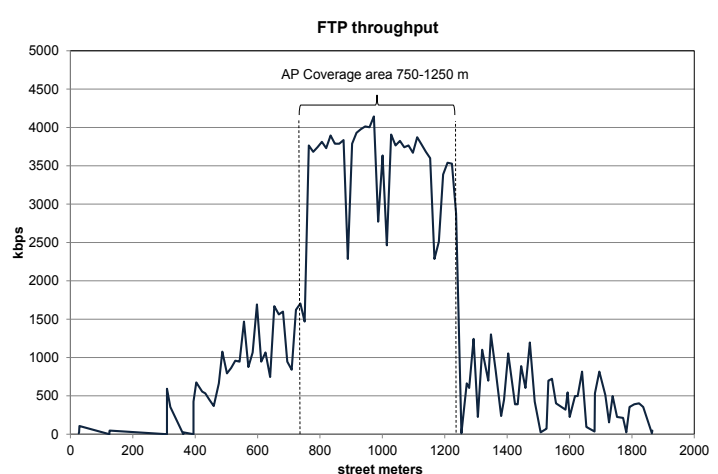

(a)

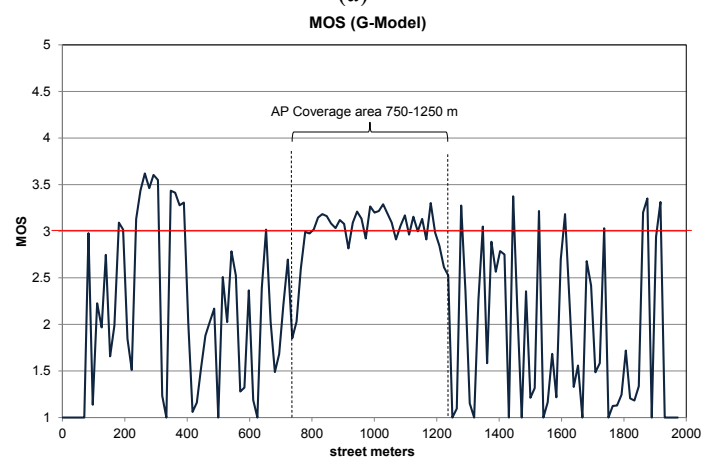

(b)

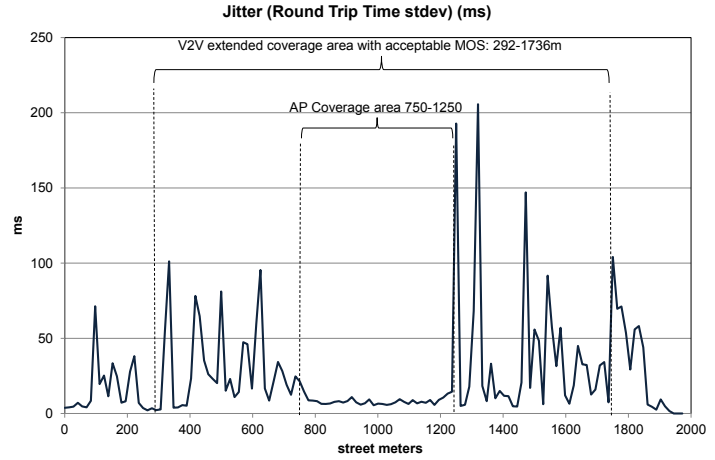

(c)

Figure 7. FPS and FTP competence: a) Throughput of the FTP download; b) MOS of the FPS game; c) Jitter of the FPS game

quality inside the car. On the other hand, tests with FTP background traffic show the need for mechanisms limiting its bandwidth, as its presence heavily deteriorates the quality of FPS games.

As future work, we want to extend the study to the situation in which different numbers of cars are running the game. In addition, we plan to build an agent that, exploiting the contact times that may occur between a car and an AP, offers a player different types of gaming experiences depending on the network status.

\section{REFERENCES}

[1] J. Manweiler, S. Agarwal, M. Zhang, R. Roy Choudhury, and P. Bahl, "Switchboard: a matchmaking system for multiplayer mobile games". In Proceedings 9th international conf. Mobile systems, applications, and services (MobiSys '11). ACM, New York, USA, pp. 71-84, 2011.

[2] J. Ott, D. Kutscher, "Drive-thru Internet: IEEE $802.11 \mathrm{~b}$ for "automobile" users". 23rd Annual IEEE Conference on Computer Communications (INFOCOM), 2004
[3] J. Eriksson, H. Balakrishnan, and S. Madden, "Cabernet: vehicular content delivery using WiFi". In Proceedings of the 14th ACM international conference on Mobile computing and networking (MobiCom '08). ACM, New York, NY, USA, 199-210, 2008.

[4] V. Namboodiri, M. Agarwal, L. Gao, "A study on the feasibility of mobile gateways for vehicular ad-hoc networks," Proc. 1st International Workshop on Vehicular Ad Hoc Networks, pp.66-75, 2004.

[5] G. Marfia, A. Amoroso, M. Roccetti, "On the Design and Run of VANET Road Experiments"'. Proc. 1st IEEE International Workshop on Vehicular Communications and Applications (VCA'12) - 11th Annual Mediterranean Ad Hoc Networking Conference (MEDHOCNET'12), Cyprus, IEEE, June 2012.

[6] R. Gass, J. Scott, and C. Diot. Measurements of In-Motion 802.11 Networking. In Proceedings of the Seventh IEEE Workshop on Mobile Computing Systems Applications (WMCSA '06). IEEE Computer Society, Washington, DC, USA, 69-74, 2006.

[7] C. Chambers, W. Feng, S. Sahu, D. Saha, "Measurement-based Characterization of a Collection of On-line Games". In Proceedings of the $5^{\text {th }}$ ACM SIGCOM conference on Internet Measurement (IMC'05). USENIX Association, Berkeley, 2005.

[8] S. Ratti, B. Hariri, S. Shirmohammadi, "A Survey of First-Person Shooter Gaming Traffic on the Internet". IEEE Internet Computing, pp. 60-69, September/October 2010.

[9] J.P. Singh, N. Bambos, B. Srinivasan, D. Clawin, "Wireless LAN performance under varied stress conditions in vehicular traffic scenarios". In Proceedings 56th Vehicular Technology Conference, IEEE , vol.2, pp. 743- 747, 2002.

[10] K. Shafiee, A. Attar, V.C.M. Leung, "Optimal Distributed Vertical Handoff Strategies in Vehicular Heterogeneous Networks". Sel. Areas in Communications, IEEE Journal, vol.29, no.3, pp.534-544, Mar 2011.

[11] G. Korkmaz, E. Ekici, F. Özgüner, Ü. Özgüner, "Urban multi-hop broadcast protocol for inter-vehicle communication systems". In Proceedings of the 1st ACM international workshop on Vehicular ad hoc networks (VANET '04). ACM, New York, NY, USA, 76-85, 2004.

[12] "The E-model, a computational model for use in transmission planning", ITU-T Recommendation G.107, 2011.

[13] A. F. Wattimena, R. E. Kooij, J. M. van Vugt, O. K. Ahmed, "Predicting the perceived quality of a first person shooter: the Quake IV G-model". In Proc. 5th SIGCOMM workshop Network and system support for games (NetGames '06), ACM, New York, NY, USA, 2006.

[14] S. Zander, G. Armitage, "Empirically Measuring the QoS Sensitivity of Interactive Online Game Players". In Proc. Australian Telecommunications Networks \& Applications Conference (ATNAC 2004), Sydney, Australia, Dec. 2004.

[15] A. P. Masucci, D. Smith, A. Crooks, M. Batty, "Random planar graphs and the London street network". The European Physical Journal B Condensed Matter and Complex Systems, Springer Berlin / Heidelberg. Vol. 71, n. 2, pp. 259-271, 2009.

[16] L. Stewart, P. Branch, Quake4, Map: q4dm1, 5players, 20Jul2006. Centre for Advanced Internet Architectures SONG Database, http://caia.swin.edu.au/sitcrc/song/download_traces_full.html\#q4

[17] M. Dick, O. Wellnitz, L. Wolf, "Analysis of factors affecting players' performance and perception in multiplayer games". In Proc. 4th ACM SIGCOMM workshop on Network and system support for games (NetGames '05). ACM, New York, NY, USA, 1-7, 2005.

[18] K. Zhu, D. Niyato, P. Wang, E. Hossain, D. I. Kim, "Mobility and handoff management in vehicular networks: a survey". Wirel. Commun. Mob. Comput. Volume 11, Issue 4 pp. 459-476. April 2011.

[19] F. Li, Y, Wang, "Routing in vehicular ad hoc networks: A survey". IEEE Vehicular Technology Magazine, vol.2, no.2, pp.12-22, June 2007.

[20] G. Marfia, M. Roccetti, "Vehicular Congestion Detection and ShortTerm Forecasting: A New Model with Results". IEEE Transactions on Vehicular Technology, IEEE Vehicular Technology Society, Vol. 60, n. 7, pp. 2936-2948, 2011.

[21] Claudio E. Palazzi, Stefano Ferretti, Marco Roccetti, "Smart Access Points on the road for online gaming in vehicular networks". Entertainment Computing, Volume 1, Issue 1, pp 17-26, January 2009. 